\title{
Características clínicas de los pacientes con nutrición parenteral en el Hospital de Especialidades Pediátricas Omar Torrijos Herrera, Panamá
}

\author{
Clinical characteristics of patients with parenteral nutrition at the Pediatric \\ Specialities Hospital Omar Torrijos Herrera, Panamá \\ Características clínicas de pacientes com nutrição parenteral no Hospital das \\ Especialidades Pediátricas Omar Torrijos Herrera, Panamá
}

Esther Soto Gómez' , Judith Ho Urriola1*

Recibido: 13 de febrero de 2019. Aceptado para publicación: 29 de julio de 2019.

Publicado en línea: 9 de noviembre de 2019

https://doi.org/10.35454/rncm.v3n1.023

\section{Resumen}

Introducción: el paciente pediátrico se caracteriza por ser más sensible a las restricciones energéticas y de nutrientes. Los equipos de soporte nutricional han optimizado la práctica nutricional intrahospitalaria.

Objetivo: describir las características clínicas de los pacientes con nutrición parenteral en el Hospital de Especialidades Pediátricas Omar Torrijos Herrera, entre 2008 y 2016.

Metodología: estudio descriptivo retrospectivo, no experimental, para el cual se utilizó la base de datos del Equipo de Soporte Nutricional (2008 - 2016). Se utilizó el programa Epi Info 7.2.0.1 para crear la base de datos y su análisis, calculando frecuencia, distribuciones, y medidas de resumen y dispersión.

Resultados: en el estudio se incluyeron 416 pacientes. El grupo etario de mayor frecuencia fue el de los escolares (30\%), con un promedio de edad y desviación estándar de 5,7 años \pm 5,0 años. El 62,5\% era de sexo masculino. La principal indicación de nutrición parenteral fue la cirugía gastrointestinal $(46,4 \%$ ) y la apendicitis complicada perforada fue el diagnóstico más frecuente. El intervalo de duración de uso de 1 - 7 días se presentó con mayor frecuencia $(61,1 \%)$.

\section{Summary}

Introduction: The pediatric patient is characterized by being more sensitive to energy and nutrient restrictions. Nutritional support teams have optimized in-hospital nutritional practice.

Objective: To describe the clinical characteristics of patients with parenteral nutrition at the Pediatric Specialties Hospital Omar Torrijos Herrera, since 2008 to 2016.

Methodology: a retrospective, nonexperimental descriptive study using the Nutrition Support Team database (2008 - 2016). The Epi Info 7.2.0.1 program was used to create the database and its analysis, calculating frequency distributions and measures of summary and dispersion.

Results: 416 patients were included in the study. The most frequent age group were school children (30\%), with an average age and standard deviation of 5.7 years \pm 5.0 years. $62.5 \%$ were male. The main indication for parenteral nutrition was gastrointestinal surgery (46.4\%) and complicated perforated appendicitis was the most frequent diagnosis. The interval of duration of use of 0-7 days was presented with greater frequency (61.1\%).

Conclusion: The use of protocols and clinical guidelines is necessary to

\section{Resumo}

Introdução: o paciente pediátrico é caracterizado por ser mais sensível às restrições energéticas e de nutrientes. As equipes de suporte nutricional otimizaram a prática nutricional intra-hospitalar.

Objetivo: descrever as características clínicas dos pacientes com nutriçao parenteral no Hospital de Especialidades Pediátricas Omar Torrijos Herrera, entre 2008 e 2016.

Metodologia: estudo descritivo retrospectivo, não experimental, para o qual foi utilizado o banco de dados da Equipe de Suporte Nutricional (2008 - 2016). O programa Epi Info 7.2.0.1 foi utilizado para criar o banco de dados e sua análise, calculando frequência, distribuição e medidas de resumo e dispersão.

Resultados: 416 pacientes foram incluídos no estudo. A faixa etária mais frequente foi de escolares ( $30 \%)$, com média de idade e desvio padrão de 5,7 anos \pm 5,0 anos, $62,5 \%$ eram do sexo masculino. A principal indicação de nutrição parenteral foi a cirurgia gastrointestinal $(46,4 \%)$ e a apendicite complicada perfurada foi o diagnóstico mais frequente. A duração de uso de 1 - 7 dias for apresentada com maior frequência $(61,1 \%)$. 
Conclusión: La utilización de protocolos y guías clínicas es necesaria para establecer las indicaciones, requerimientos y seguimiento de la nutrición parenteral.

Palabras clave: nutrición parenteral, soporte nutricional, malnutrición infantil. establish the indications, requirements and monitoring of parenteral nutrition.

Keywords: Parenteral nutrition; Nutritional support; Child malnutrition.
Conclusão: O uso de protocolos clínicos e diretrizes é necessário para estabelecer as indicações, requisitos e monitorização da nutrição parenteral.

Palavras-chave: nutrição parenteral, suporte nutricional, desnutrição infantil.
Hospital de Especialidades Pediátricas Omar Torrijos Herrera, Vía España, Calle Lucas Zarak, Corregimiento de Pueblo Nuevo, Ciudad de Panamá, República de Panamá
*Correspondencia: Judith Ho Urriola doctoraho@hotmail.com

\section{INTRODUCCIÓN}

La desnutrición hospitalaria es un grave problema que afecta a un importante número de pacientes hospitalizados, puede resultar en retraso en la recuperación del paciente, mayor susceptibilidad a infecciones, prolongación del tiempo de estancia hospitalaria y aumento del número de reingresos, lo que redunda en aumento de la morbimortalidad y costo sanitario ${ }^{(1)}$. La Sociedad Europea de Gastroenterología, Hepatología y Nutrición Pediátrica (European Society for Pediatric Gastroenterology Hepatology and Nutrition, ESPGHAN) en 2005 reportó que la prevalencia de malnutrición en los hospitales pediátricos oscila entre $15 \%$ y $30 \%$, dependiendo de los criterios utilizados para definir la malnutrición, la población de pacientes y su espectro de enfermedad ${ }^{(2)}$. A pesar de los grandes avances en la calidad de la atención, la prevalencia de malnutrición en los niños hospitalizados no ha disminuido en los últimos 20 años.

El paciente pediátrico críticamente enfermo presenta condiciones que no permiten una buena correlación energética y nutricional, por lo que es imprescindible aplicar medidas eficaces de soporte nutricional ${ }^{(3)}$.

La nutrición parenteral es una herramienta imprescindible en el apoyo nutricional de pacientes. Tiene múltiples indicaciones, siendo utilizada en niños desnutridos o con riesgo de desnutrición secundario a una patología digestiva o no digestiva, y promueve el crecimiento en enfermedades que cursan con afectación grave de la función intestinal en las primeras etapas de la vida.

El papel de los equipos multidisciplinarios de soporte nutricional intrahospitalario, utilizando protocolos estandarizados, ha sido evaluado en múltiples estudios, reportándose optimización de las prácticas nutricio- nales, como el incremento del uso de nutrición enteral entre $25 \%$ y $67 \%$, disminuyendo en forma significativa la utilización de nutrición parenteral, lo que puede representar menor riesgo de muerte (hasta $83 \%$ ) en los niños que recibieron nutrición enteral ${ }^{(4)}$. La disminución del uso inadecuado de la nutrición parenteral ha conducido a ahorro de costos luego de la implementación de los grupos de soporte nutricional y la intervención de enfermeras especializadas en soporte nutricional reduce notablemente la infección asociada al catéter venoso. Se ha demostrado que a través de un equipo de soporte nutricional, el uso inadecuado de nutrición parenteral puede disminuir entre $16,5 \%$ y $8,9 \%$, siguiendo lineamientos de soporte nutricional basados en evidencia ${ }^{(5)}$.

No existen datos nacionales publicados acerca del uso de nutrición parenteral en pacientes pediátricos, por lo que el objetivo de este estudio es describir las características clínicas y epidemiológicas de los pacientes pediátricos que recibieron nutrición parenteral en el Hospital de Especialidades Pediátricas de Panamá.

\section{METODOLOGÍA}

Se realizó un estudio descriptivo, transversal y retrospectivo en el Hospital de Especialidades Pediátricas Omar Torrijos Herrera, centro de referencia de atención pediátrica (edades de 0 a 15 años) con seguridad social, ubicado en el corregimiento de Pueblo Nuevo, Vía España, Ciudad de Panamá, con 129 camas disponibles. Se incluyeron todos los pacientes de todas las edades, sexo y patologías, hospitalizados de enero de 2008 a diciembre de 2016, a quienes se les prescribió nutrición parenteral como indicaba la base de datos del Equipo de Soporte nutricional (486 pacientes). 
Se excluyeron pacientes con datos incompletos, y un paciente con nutrición parenteral domiciliaria, resultando una muestra final de 416 pacientes.

Se creó un formulario para la recolección de datos donde las variables estudiadas fueron: edad, la cual se dividió en los grupos etarios recién nacidos (0 - 28 días), lactantes menores (29 días a 11 meses), lactantes mayores ( 12 meses - 1 año, 11 meses 29 días), preescolares ( 2 - 5 años), escolares (6 - 11 años) y adolescentes (12 años o más); sexo, diagnóstico, duración en días de la nutrición parenteral, servicio hospitalario solicitante de la nutrición parenteral, y vía de administración (central o periférica).

Se utilizó el programa Epi Info 7.2.0.1 para el análisis de la misma, calculando distribuciones de frecuencia (porcentajes), medidas de resumen y dispersión (media, mediana, moda y desviación estándar). Por medio de la medida de Curtosis se evaluó si los datos cuantitativos presentaban una distribución normal. En cuanto a la duración de la nutrición parenteral esta presentó una distribución no normal por lo cual se evaluaron la mediana y la distribución por intervalos.

\section{Aspectos éticos de la investigación}

El estudio fue aprobado por el Comité Nacional de Ética. Se aceptó cumplir con los principios éticos y morales que deben regir toda investigación que involucra sujetos humanos como lo son: Declaración de Helsinki, Buenas Prácticas Clínicas y las Normas y criterios éticos establecidos en los códigos nacionales de ética y leyes vigentes.

\section{RESULTADOS}

Los pacientes fueron clasificados por grupo etario donde $6 \%(25 / 416)$ correspondió a recién nacidos, $24 \%(99 / 416)$ a lactantes menores, $6 \%(27 / 416)$ a lactantes mayores, $16 \%(67 / 416)$ a preescolares, $30 \%$ $(124 / 416)$ a escolares y $18 \%(74 / 416)$ a adolescentes.

El promedio de edad fue 5,7 años \pm 5 años, $62,5 \%$ (260/416) de los pacientes fueron de sexo masculino. $\mathrm{Al}$ ser un centro de referencia pediátrico, muchos pacientes con patologías crónicas continúan seguimiento con especialistas de la institución a pesar de que sean mayores de 15 años, lo que corresponde al caso especial de un paciente de 20 años.

Se registraron 115 diagnósticos de los cuales 65,1\% (271/416) correspondieron a patologías de origen digestivo y $34,9 \%(145 / 416)$ a patologías de origen no digestivo. Dentro de las patologías de origen digestivo se destacan apendicitis complicada perforada, la más frecuente de este grupo con $18 \%$ (49/271) de los casos, malformaciones gastrointestinales, pancreatitis aguda, perforaciones gastrointestinales y enfermedad inflamatoria intestinal, entre otras. Entre las patologías de origen no digestivo se pueden mencionar: cardiopatías, enfermedades oncológicas, desnutrición proteico-calórica, cirugías no gastrointestinales y sepsis, que es la más frecuente de este grupo con 14,8 \% (21/145).

Las indicaciones de nutrición parenteral se muestran en la Tabla 1.

Tabla 1. Indicaciones de nutrición parenteral en el Hospital de Especialidades Pediátricas Omar Torrijos Herrera, Panamá (2008-2016)

\begin{tabular}{|l|c|}
\hline \multicolumn{1}{|c|}{ Indicación } & \multicolumn{1}{|c|}{$\begin{array}{c}\text { Cantidad de } \\
\text { pacientes }\end{array}$} \\
\hline Cirugía gastrointestinal & $193(46 \%)$ \\
\hline Gastrointestinal no quirúrgica & $72(17 \%)$ \\
\hline Oncológico & $33(8 \%)$ \\
\hline Respiratorio & $25(6 \%)$ \\
\hline Infeccioso & $22(5 \%)$ \\
\hline Cardíaco & $20(5 \%)$ \\
\hline Trauma múltiple & $4(1 \%)$ \\
\hline Trasplante & $1(1 \%)$ \\
\hline Otro & $46(11 \%)$ \\
\hline Total & $416(100 \%)$ \\
\hline
\end{tabular}

Fuente: Base de datos. Hospital de Especialidades Pediátricas Omar Torrijos Herrera. Caja de Seguro Social. Panamá 2008-2016.

La apendicitis complicada perforada se presentó en mayor proporción en el grupo etario de escolares con $42,9 \%(21 / 49)$, seguido de preescolares con $36,7 \%$ $(18 / 49)$ y adolescentes $20,4 \%(10 / 49)$. Por otra parte, la cardiopatía congénita se presentó con mayor frecuencia en el grupo de lactantes menores con $55 \%(6 / 20)$ de los casos, seguido del grupo de recién nacidos con $30 \%$ $(6 / 20)$, presentándose en menor proporción en el grupo de escolares con $10 \%(2 / 20)$ y lactantes mayores $5 \%$ $(1 / 20)$. La totalidad de los casos de invaginación intestinal se presentó en el grupo de lactantes menores (Figura 1).

La mediana de tiempo de la nutrición parenteral fue de 6 días. El 61,1 \% de los pacientes (254/416) tuvieron entre 1 y 7 días de nutrición parenteral (NP), 23,1\% (96/416) de 8 - 15 días, 10,3\%(43/416) de 16 - 29 días y $5 \%(21 / 416)$ de $1-3$ meses y $0,5 \%(2 / 416)$ de 
4 - 6 meses, ningún paciente tuvo la NP por más de 7 meses (Figura 2).

La Tabla 2 muestra que las patologías más frecuentes mantuvieron una duración de nutrición parenteral de 1 - 7 días: 73,5\% (36/49) de los casos de apendicitis complicada, $83,8 \%(31 / 37)$ de apendicitis aguda, $50 \%$ $(5 / 10)$ de plastrón apendicular, $65 \%(13 / 20)$ de cardiopatía congénita, $80 \%(8 / 10)$ de invaginación intestinal, 54,5\%(12/22) de neumonía, 73,7 \% de obstrucción intestinal y $57 \%(12 / 21)$ de sepsis/shock séptico. Por otra parte, dentro de los diagnósticos más frecuentes, la pancreatitis aguda fue la que se presentó en mayor proporción con un intervalo de duración de 8 - 15 días en $41,7 \%$ de los casos (5/12).

Los servicios que solicitaron nutrición parenteral en orden de frecuencia fueron: Cirugía Pediátrica $50 \%$ (208/416), Cuidados Intensivos 33 \% (137/416), Medicina Pediátrica 8 \% (33/416), Hematooncología 2,6 \% (11/416), Gastroenterología 2,6 \% (11/416), Neumología 0,5 \% (2/416). Otros servicios representan $3,1 \%$ de los casos (13/416), dentro de los que encontramos Neurocirugía, Urología, Cardiología e Infectología.

Según la vía de administración, 97,4 \% (405/416) de las nutriciones parenterales se administraron por vía

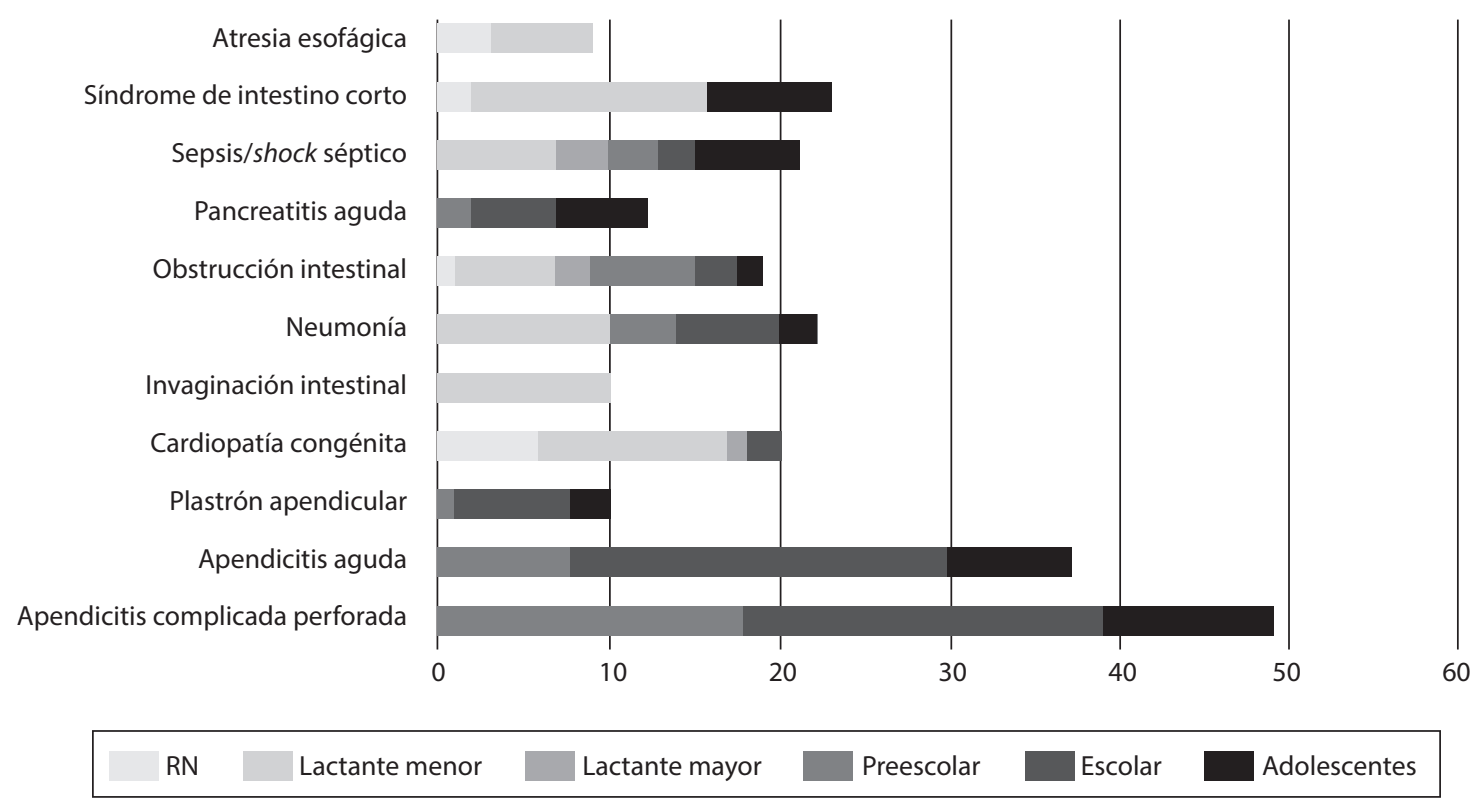

Figura 1. Diagnósticos más frecuentes según grupo etario. Las barras muestran número de pacientes. Hospital de Especialidades Pediátricas Omar Torrijos Herrera. Panamá (2008 - 2016). Fuente: Base de datos. Hospital de Especialidades Pediátricas Omar Torrijos Herrera. Caja de Seguro Social. Panamá 2008 - 2016.

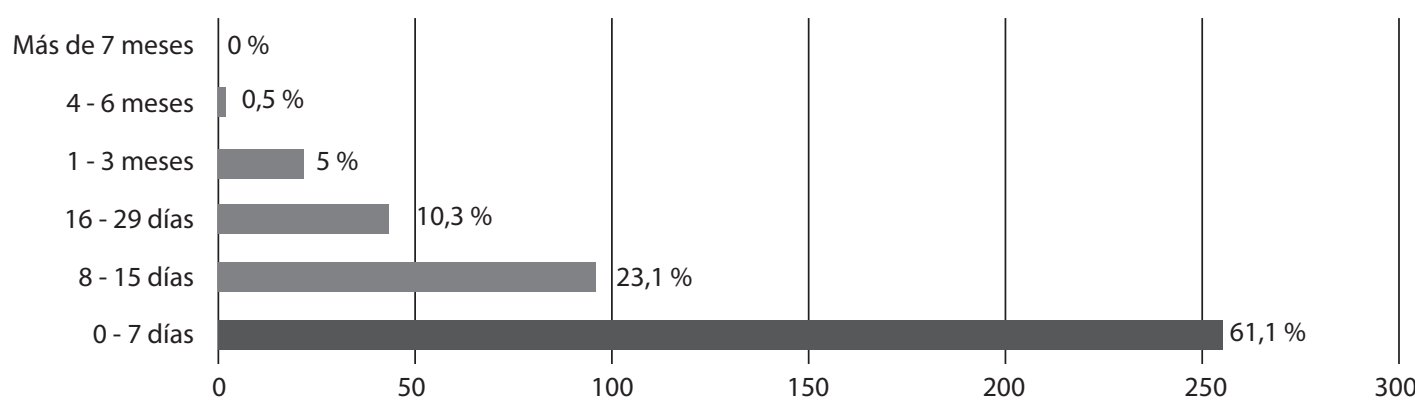

Figura 2. Tiempo con nutrición parenteral según grupo etario. Hospital de Especialidades Pediátricas Omar Torrijos Herrera, Panamá (2008-2016). Fuente: Base de datos. Hospital de Especialidades Pediátricas Omar Torrijos Herrera. Caja de Seguro Social. Panamá (20082016). 
Tabla 2. Diagnósticos más frecuentes y duración de la nutrición parenteral. Hospital de Especialidades Pediátricas Omar Torrijos Herrera, Panamá (2008 - 2016)

\begin{tabular}{|c|c|c|c|c|c|c|c|}
\hline \multirow[t]{2}{*}{ Diagnóstico } & \multicolumn{7}{|c|}{ Cantidad de pacientes según intervalos de duración } \\
\hline & 0 - 7 días & 8 - 15 días & 16 - 29 días & $1-3$ meses & 4 - 6 meses & Más de 7 meses & Total \\
\hline $\begin{array}{l}\text { Apendicitis complicada } \\
\text { perforada }\end{array}$ & $\begin{array}{c}36 \\
(73,5 \%)\end{array}$ & $\begin{array}{c}11 \\
(22,4 \%)\end{array}$ & $\begin{array}{c}2 \\
(4,1 \%)\end{array}$ & --- & --- & --- & $\begin{array}{c}49 \\
(100 \%)\end{array}$ \\
\hline Apendicitis aguda & $\begin{array}{c}31 \\
(83,8 \%)\end{array}$ & $\begin{array}{c}6 \\
(16,2 \%)\end{array}$ & --- & --- & --- & --- & $\begin{array}{c}37 \\
(100 \%)\end{array}$ \\
\hline Plastrón apendicular & $\begin{array}{c}5 \\
(50 \%)\end{array}$ & $\begin{array}{c}3 \\
(30 \%)\end{array}$ & & $\begin{array}{c}2 \\
(20 \%)\end{array}$ & & & $\begin{array}{c}10 \\
(100 \%)\end{array}$ \\
\hline Cardiopatía congénita & $\begin{array}{c}13 \\
(65 \%)\end{array}$ & $\begin{array}{c}4 \\
(20 \%)\end{array}$ & $\begin{array}{c}2 \\
(10 \%)\end{array}$ & $\begin{array}{c}1 \\
(5 \%)\end{array}$ & --- & --- & $\begin{array}{c}20 \\
(100 \%)\end{array}$ \\
\hline Invaginación intestinal & $\begin{array}{c}8 \\
(80 \%)\end{array}$ & $\begin{array}{c}1 \\
(10 \%)\end{array}$ & $\begin{array}{c}1 \\
(10 \%)\end{array}$ & --- & --- & --- & $\begin{array}{c}10 \\
(100 \%)\end{array}$ \\
\hline Neumonía & $\begin{array}{c}12 \\
(54,5 \%)\end{array}$ & $\begin{array}{c}7 \\
(31.9 \%)\end{array}$ & $\begin{array}{c}2 \\
(9,1 \%)\end{array}$ & $\begin{array}{c}1 \\
(4,5 \%)\end{array}$ & --- & --- & $\begin{array}{c}22 \\
(100 \%)\end{array}$ \\
\hline Obstrucción intestinal & $\begin{array}{c}14 \\
(73,7 \%)\end{array}$ & $\begin{array}{c}4 \\
(21 \%)\end{array}$ & $\begin{array}{c}1 \\
(5 \%)\end{array}$ & --- & --- & --- & $\begin{array}{c}19 \\
(100 \%)\end{array}$ \\
\hline Pancreatitis aguda & $\begin{array}{c}4 \\
(33,3 \%)\end{array}$ & $\begin{array}{c}5 \\
(41,7 \%)\end{array}$ & $\begin{array}{c}3 \\
(25 \%)\end{array}$ & --- & --- & --- & $\begin{array}{c}12 \\
(100 \%)\end{array}$ \\
\hline Sepsis / shock séptico & $\begin{array}{c}12 \\
(57 \%)\end{array}$ & $\begin{array}{c}5 \\
(24 \%)\end{array}$ & $\begin{array}{c}2 \\
(9,5 \%)\end{array}$ & $\begin{array}{c}2 \\
(9,5 \%)\end{array}$ & --- & --- & $\begin{array}{c}21 \\
(100 \%)\end{array}$ \\
\hline Síndrome de intestino corto & $\begin{array}{c}7 \\
(30,4 \%)\end{array}$ & $\begin{array}{c}7 \\
(30,4 \%)\end{array}$ & $\begin{array}{c}4 \\
(17,4 \%)\end{array}$ & $\begin{array}{c}4 \\
(17,4 \%)\end{array}$ & $\begin{array}{c}1 \\
(4,4 \%)\end{array}$ & --- & $\begin{array}{c}23 \\
(100 \%)\end{array}$ \\
\hline Atresia esofágica & $\begin{array}{c}3 \\
(33 \%)\end{array}$ & $\begin{array}{c}2 \\
(22 \%)\end{array}$ & $\begin{array}{c}3 \\
(33 \%)\end{array}$ & $\begin{array}{c}1 \\
(11 \%)\end{array}$ & --- & --- & $\begin{array}{c}9 \\
(100 \%)\end{array}$ \\
\hline
\end{tabular}

Fuente: Base de datos. Hospital de Especialidades Pediátricas Omar Torrijos Herrera. Caja de Seguro Social. Panamá (2008 - 2016)

central y $2,6 \%(11 / 416)$ por vía periférica. El promedio de pacientes que utilizaron nutrición parenteral por año fue 46 (rango 27 - 59).

\section{DISCUSIÓN}

El objetivo del presente estudio fue describir las características clínicas y epidemiológicas de los pacientes pediátricos que recibieron nutrición parenteral en el Hospital de Especialidades Pediátricas de Panamá. Se evaluaron 416 pacientes pediátricos que recibieron nutrición parenteral entre 2008 y 2016. Este grupo se caracterizó por recibir en su mayoría NP por vía central (97\%), con una media de 6 días, por indicaciones secundarias a patología digestiva $(65,1 \%)$ y en la mitad de los casos por interconsulta del Servicio de Cirugía.
Recibieron NP niños de todas las edades principalmente lactantes $(24 \%)$, escolares $(30 \%)$ y de sexo masculino $(62,5 \%)$.

Detallar esta población es importante por el impacto que el soporte nutricional tiene en la evolución y recuperación del paciente pediátrico, para garantizar un tratamiento nutricional adecuado a través del Equipo de Terapia Nutricional multidisciplinario del Hospital de Especialidades Pediátricas de Panamá. Este equipo, creado en el año 2003, funciona como una unidad de apoyo que se encarga de la evaluación, formulación, preparación, administración, cuidados y seguimiento del paciente pediátrico hospitalizado. Se ha demostrado que cuando los equipos de soporte nutricional participan en el cuidado de pacientes que necesitan 
apoyo nutricional se disminuye el uso inadecuado y a corto plazo, costos y complicaciones que se puedan derivar de la nutrición parenteral ${ }^{(6)}$.

Por grupo etario, esta muestra contó con pocos recién nacidos $(6 \%)$, cifra que contrasta con otros estudios $(47,8 \%)^{(7)}$, lo que puede explicarse por el hecho de que este hospital no cuenta con sala de neonatología.

Nuestro estudio identificó las indicaciones de nutrición parenteral según patologías digestivas o no digestivas, tal y como lo recomienda la Sociedad Española de Pediatría $(2010)^{(8)}$. En nuestro estudio la principal indicación de nutrición parenteral fue la cirugía gastrointestinal (46,4\%), siendo la apendicitis complicada perforada el diagnóstico más frecuente $(11,8 \%)$. Esto concuerda con el estudio de Moreno Villares et al. ${ }^{(9)}$ en donde las intervenciones quirúrgicas sobre el aparato digestivo fueron la indicación más frecuente en los niños mayores de 2 años.

Diferentes guías de nutrición parenteral como las de la Sociedad Española de Nutrición Parenteral y Enteral (SENPE), ESPGHAN y la Sociedad Europea de Nutrición Clínica (ESPEN) ${ }^{(10)}$ establecen que la nutrición parenteral se debe indicar para prevenir o corregir los efectos adversos de la malnutrición en pacientes que no pueden obtener aportes suficientes por vía oral o enteral, requieran ayuno absoluto por un tiempo superior o igual a 5 días, o en pacientes pediátricos donde no es posible la vía enteral, y reciben menos de $50 \%$ de sus necesidades durante 7 días o antes si el paciente está previamente malnutrido y que debe mantenerse hasta cuando se consiga una adecuada transición a la nutrición enteral y que dichos aportes alcancen al menos $2 / 3$ de los requerimientos nutricionales estimados. En nuestro estudio, el intervalo de duración de la nutrición parenteral fue de 1 - 7 días en $61,1 \%$ de los casos y la mediana fue de 6 días.

En cuanto a los servicios que solicitaron nutrición parenteral, $50 \%$ de los casos corresponde al servicio de cirugía pediátrica, lo que concuerda con el estudio de Mantegazza et al. ${ }^{(11)}$, en el que $35 \%$ fue indicada por servicios quirúrgicos, mientras que las especialidades médicas, incluyendo oncología, indicaron nutrición parenteral en $34 \%$ de los casos. Sin embargo, en nuestro estudio solo $13,7 \%$ de las nutriciones parenterales fueron solicitadas por servicios de especialidades no quirúrgicas como Medicina Pediátrica, Gastroenterología, Hematooncología, Neumología y Nefrología. Esto lleva a plantearnos si la nutrición parenteral estará siendo subutilizada por las especialidades médicas, ya que en diferentes estudios se observan patologías, en especial las hematooncológicas como las más fre- cuentes de indicación de nutrición parenteral y en este estudio solo representó $2,6 \%$ de los casos, por lo que se requiere continuar estudios donde se analicen las indicaciones de nutrición parenteral de manera más detallada en nuestro hospital.

En el presente estudio la vía de administración de nutrición parenteral más utilizada fue la central (97,4\%). Esto se debe a que la vía periférica en nuestra institución solo está indicada como medida temporal y cuando los requerimientos y la osmolaridad de la mezcla parenteral son bajos.

La principal limitante de este estudio fue que, al ser un estudio retrospectivo no nos permitió establecer una secuencia temporal de las variables estudiadas, tener mayor riesgo de sesgo por la falta de datos disponibles o confiables por lo que el análisis de los datos y la falta de estudios previos en nuestro país pudieron afectarse.

\section{CONCLUSIONES}

Este estudio permitió conocer las características clínicas de los pacientes que recibieron nutrición parenteral en el Hospital de Especialidades Pediátricas Omar Torrijos Herrera entre los años 2008 - 2016. Los resultados concuerdan en su mayoría con los descritos en otros estudios. Es importante que a través de protocolos y guías clínicas se establezcan las indicaciones, requerimientos y seguimiento de la nutrición parenteral en los pacientes del Hospital de Especialidades Pediátricas para garantizar la atención y calidad que se brinda.

\section{Agradecimientos}

Al equipo de Terapia Nutricional del Hospital de Especialidades Pediátricas, en especial a la Lic. Mara de Gómez.

\section{Conflicto de intereses}

Ninguno.

\section{Financiación}

El presente artículo no tuvo financiación.

\section{Declaración de autoría}

Los autores participaron en la redacción del artículo, la concepción, la realización y el desarrollo de la investigación, la obtención de los datos, el análisis e interpretación de los resultados. De igual forma revisaron el artículo y validaron su versión final. 


\section{Referencias bibliográficas}

1. Gomis P, Gómez L, Martinez C, Moreno JM, Pedrón C, Pérez-Portabella C, et al. Documento de consenso SENPE/ SEGHNP/SEFH sobre nutrición parenteral pediátrica. Nutr Hosp. 2007;22(6):710-9.

2. Agostoni C, Axelson I, Colomb V, Goulet O, Koletzko B, Michaelsen KF, et al. The need for nutrition support teams in Pediatric Units: A Commentary by the ESPGHAN Committee on Nutrition. J Pediatr Gastroeterol Nutr. 2005; 41(2):267-71.

3. Galera-Martínez R, López-Ruzafa E, Moráis López A, Lama More RA. Actualización en el soporte nutricional del paciente pediátrico críticamente enfermo. Acta Pediátr Esp. 2017;75(7-8):e117-e23.

4. Nieva A, Capra D. Guía Práctica Clínica de Soporte Nutricional del Paciente Pediátrico Crítico. Medicina Intensiva. 2012; 29(Suplemento 1):1-15.

5. Sriram K, Cyriac T, Fogg LF. Effect of nutritional support team restructuring on the use of parenteral nutrition. Nutrition. 2010;26(7-8):735-9.

6. Infante D. Guía de Nutrición Pediátrica Hospitalaria. Hospital Universitario Materno-Infantil Vall d'Hebron, Barcelona: Editorial Ergo; 2010. p.25-44.
7. Ríos-González S, Anaya-Florez MS, Gutiérrez-Hernández JI, Morán-Villota S. Nutrición Parenteral en pacientes pediátricos: indicación y complicaciones en tercer nivel. Rev Med Inst Seguro Soc. 2015;53(S3):262-9.

8. Sociedad Española de Gastroenterología, Hepatología y Nutrición Pediátrica, Academia Española de Pediatría. Nutrición Parenteral. Protocolos diagnóstico-terapéuticos de Gastroenterología, Hepatología y Nutrición Pediátrica. Ergon 2010 [Consultado Enero 2018]. Disponible en: http:// seghnp.org

9. Moreno Villares JM, Fernández Carrión F, Sánchez Díaz JI, Gomis Muñoz P, León Sanz M. Current use of parenteral nutrition in a pediatric hospital. Comparison to the practise 8 years ago. Nutr Hosp. 2005;20(1):46-51.

10. Pedrón Giner C, Cuervas-Mons Vendrell M, Galera Martinez R, Gómez López L, Gomis Muñoz P, Irastorza Terradillos I, et al. Pediatric parenteral nutrition: clinical practice guidelines from the Spanish Society of Parenteral and Enteral Nutrition (SENPE), the Spanish Society of Pediatric Gastroenterology, Hepatology and Nutrition (SEGHNP) and the Spanish Society of Hospital Pharmacy (SEFH). Nutr Hosp. 2017;34(3):745-58.

11. Mantegazza C, Landy N, Hill SM, Zuccotti GV, Koglmeier J. Parenteral Nutrition in Hospitalized Children. Med Clin Rev. 2016;2(1):4. 